\title{
Perfil do uso e manuseio de anti-hipertensivos em indivíduos acima de 60 anos
}

\author{
Profile of the use and handling of antihypertensives in individuals over 60 years of age \\ Perfil del uso y manejo de antihipertensivos en personas mayores de 60 años
}

Recebido: 01/10/2021 | Revisado: 09/10/2021 | Aceito: 13/10/2021 | Publicado: 16/10/2021

Deborah Rito da Silva

ORCID: https://orcid.org/0000-0002-2454-1069 Universidade Anhembi-Morumbi, Brasil

E-mail: deborahrito@hotmail.com

Juliana Massucato Perez Chubaci

ORCID: https://orcid.org/0000-0001-9701-617X Universidade Anhembi-Morumbi, Brasil

E-mail: ju.chubaci@gmail.com

Guilherme Luis Toledo Barreto

ORCID: https://orcid.org/0000-0001-8358-0212

Universidade Anhembi-Morumbi, Brasil

E-mail: guitbarreto@gmail.com

Marie Jordão Khemiri

ORCID: https://orcid.org/0000-0003-4490-3984

Universidade Anhembi-Morumbi, Brasil

E-mail: mariejordao@gmail.com

Leandro Giorgetti

ORCID: https://orcid.org/0000-0003-2776-2286

Universidade Anhembi-Morumbi, Brasil

E-mail: lggiorgetti@anhembi.br

\begin{abstract}
Resumo
A hipertensão arterial é uma das doenças que mais acomete idosos, os quais pela influência de diversos fatores, como a idade e a polifarmácia, podem causar administração ou manuseio incorreto dos anti-hipertensivos. Assim, este estudo busca analisar hábitos realizados por idosos e/ou seus cuidadores no uso de anti-hipertensivos, podendo esses hábitos serem positivos ou negativos para o tratamento do idoso. Através de um questionário virtual em busca dessas informações com uma análise multivariada e um estudo transversal, foram obtidas 111 respostas, nas quais 81 dos entrevistados estão em uso de anti-hipertensivos, sendo os principais: bloqueador do receptor de angiotensina, diurético tiazídico, inibidor da enzima conversora de angiotensina e bloqueador do canal de cálcio. Verificou-se que a maioria dos pacientes faz a ingestão de forma correta com água, e poucos realizam o corte de comprimidos e a abertura de cápsulas. Referente ao armazenamento, os principais locais, de acordo com a pesquisa, são a cozinha e o quarto, e muitos idosos mantêm o medicamento na embalagem original. A pesquisa apresentou resultados positivos, mas os erros identificados poderiam ser evitados com a orientação correta e reforçada dos profissionais de saúde.

Palavras-chave: Idosos; Polimedicação; Interações medicamentosas; Administração oral de medicamentos; Antihipertensivos; Armazenamento de medicamentos.
\end{abstract}

\begin{abstract}
Hypertension is one of the diseases that most affects the elderly, which, due to the influence of several factors, such as age and polypharmacy, may cause the incorrect administration or handling of antihypertensives. Thus, this study aims to analyze the habits of the elderly and/or their caregivers regarding the use of antihypertensives, which can be positive or negative for the treatment of the elderly. Through a virtual questionnaire in search of this information with a multivariate analysis and a cross-sectional study, 111 answers were obtained, of which 81 of the interviewees are using antihypertensives, the main ones being angiotensin receptor blocker, thiazide diuretic, angiotensin-converting enzyme inhibitor, and calcium channel blocker. It was found that most patients ingest the drugs correctly with water, and few cut the tablets and open the capsules. Regarding storage, the main places according to the survey are the kitchen and the bedroom, and many elderly people keep the medication in its original packaging. The survey presented positive results, but the errors identified could be avoided with correct and reinforced guidance from health professionals.
\end{abstract}

Keywords: Elderly; Polypharmacy; Drug interactions; Oral administration of drugs; Anti-hypertensives; Drug storage.

\section{Resumen}

La hipertensión arterial es una de las enfermedades que más afectan a los ancianos, que por la influencia de diversos factores, como la edad y la polifarmacia, pueden provocar la administración o el uso incorrecto de los 
antihipertensivos. Así, este estudio pretende analizar los hábitos que realizan los ancianos y/o sus cuidadores en el uso de los antihipertensivos, pudiendo ser estos hábitos positivos o negativos para el tratamiento de los ancianos. Mediante un cuestionario virtual en busca de esta información con un análisis multivariado y un estudio transversal, se obtuvieron 111 respuestas, de las cuales 81 de los entrevistados están utilizando antihipertensivos, siendo los principales el bloqueador del receptor de angiotensina, el diurético tiazídico, el inhibidor de la enzima convertidora de angiotensina y el bloqueador de los canales de calcio. Se verificó que la mayoría de los pacientes ingieren los fármacos correctamente con agua, y pocos cortan los comprimidos o abren las cápsulas. En cuanto al almacenamiento, los principales lugares según la encuesta son la cocina y el dormitorio, y muchos ancianos guardan la medicación en su envase original. La investigación presentó resultados positivos, pero los errores identificados podrían evitarse con una orientación correcta y reforzada por parte de los profesionales sanitarios.

Palabras clave: Ancianos; Polifarmacia; Interacciones medicamentosas; Administración oral de medicamentos; Antihipertensivos; Almacenamiento de medicamentos.

\section{Introdução}

Existem diversas doenças que acometem a população idosa, sendo a hipertensão arterial uma das mais comuns. De acordo com as Diretrizes Brasileiras de Hipertensão Arterial (Malachias et al., 2016), no Brasil, a Hipertensão Arterial (HA) afeta 36 milhões de indivíduos adultos, sendo mais de $60 \%$ idosos, contribuindo para $50 \%$ das mortes por doenças cardiovasculares. A HA é uma condição clínica multifatorial, a qual é caracterizada pela elevação sustentada dos níveis pressóricos, podendo ser agravada pela presença de fatores de risco, como sexo masculino, negro, idade igual ou superior a 60 anos e comorbidades como obesidade, sedentarismo, histórico familiar de doenças cardiovasculares, dislipidemia, intolerância à glicose e diabetes mellitus (DM).

A hipertensão é uma doença considerada silenciosa, sendo o diagnóstico precoce essencial para diminuir possíveis complicações, ocasionado em um tratamento ideal de forma mais rápida (Nobre de Menezes et al., 2016). Um estudo transversal de base populacional realizado com 912 idosos em 2019, mostra a prevalência de 74,9\% de idosos com hipertensão arterial (Sousa et al., 2019). Esses dados demonstram um número de casos elevado, que muitas vezes resulta em necessidade de tratamento farmacológico, que nem sempre é feito adequadamente.

Além da hipertensão, é comum notarmos uma ou mais doenças crônicas em idosos, havendo assim a necessidade de outras terapias medicamentosas em conjunto, resultando na maioria das vezes em polifarmácia. Além disso, é frequente ver um idoso sendo acompanhado por mais de um médico, o que pode resultar também em interações medicamentosas, devido à falta de comunicação. A orientação pelo médico ou farmacêutico é essencial, mas pode acabar sendo falha, resultando assim em erros de administração ou armazenamento (Burnier et al., 2020).

Um problema recorrente do armazenamento, por exemplo, é utilizar organizadores de comprimidos. Estes, ainda que possam facilitar o dia a dia, principalmente em pacientes idosos, criam a possibilidade de prejudicar a estabilidade do medicamento retirando-o da embalagem original, produzida com as condições necessárias de armazenamento, além de fazer com que o paciente confunda os medicamentos, suas datas de validade e dosagens, já que não possuem mais embalagem e identificação (Borja-Oliveira 2013).

Como mencionado anteriormente, é comum a presença de outras comorbidades em idosos, acarretando na prática da polifarmácia. A fim de diminuir custos, dificuldade na deglutição e flexibilizar a dose, por vezes orientada por um profissional de saúde, acaba sendo realizado a partição de comprimidos e/ou a abertura de cápsulas. Porém nem todos os comprimidos podem ser partidos e cápsulas abertas, pois podem prejudicar a ação do fármaco e/ou prejudicar a estabilidade do medicamento, sendo necessário consultar um médico ou farmacêutico quando necessário (Gill et al., 2012).

Outro erro relacionado é a administração de medicamentos junto a comidas, bebidas ou outros medicamentos, que podem resultar em uma interação droga-nutriente ou interação medicamentosa. Essa interação ocorre quando há qualquer alteração farmacocinética ou farmacodinâmica, seja por alimentos, bebidas ou hábitos (como por exemplo o tabagismo), que podem alterar a eficácia e efeitos adversos de alguns hipertensivos comumente utilizados, como diuréticos, inibidores da 
enzima conversora de angiotensina, bloqueadores dos receptores de angiotensina II, entre outros (Jáuregui-Garrido e JáureguiLobera, 2012).

Por meio deste estudo busca-se localizar os principais erros que idosos cometem na administração, no manuseio e no armazenamento de anti-hipertensivos a fim de demonstrar as consequências desses erros e assim poder corrigi-los.

\section{Metodologia}

Foi aplicado de forma virtual, um questionário (via Google Forms) para levantar informações sobre a administração de anti-hipertensivos em idosos, armazenamento e uso de outras medicações. Através de uma análise quantitativa (Nascimento e Cavalcante, 2018), observamos os dados de uma população idosa usuária de anti-hipertensivos a fim de agrupar as informações obtidas através do questionário e comparar com a pesquisa bibliográfica encontrada.

O questionário é composto de 6 seções: termo de consentimento esclarecido, informações gerais, comprimidos, cápsulas, armazenamento e informações adicionais. Cada seção complementa a anterior para que seja possível a coleta de dados adequados para avaliação do uso de anti-hipertensivos por idosos. O questionário pode ser respondido pelo idoso com acesso ao ambiente virtual ou por algum familiar, cuidador ou qualquer conhecido que tenha acesso às informações necessárias para completar as respostas.

Coletou-se informações de faixa etária, sexo, quantidade de medicamentos e quais são utilizados, como é realizada a ingestão, o manuseio e o armazenamento dos comprimidos e cápsulas. O questionário foi divulgado de 25/05/2021 a 01/08/2021. Com os resultados apresentados na pesquisa, verificamos a frequência de ocorrência dos principais pontos para melhorias nas orientações que os profissionais de saúde devem conceder aos pacientes idosos ou aos seus cuidadores.

Esse estudo foi aprovado pelo Comitê de Ética em Pesquisa (CEP) da Universidade Anhembi Morumbi, sob o parecer número 4.694.926, tendo os participantes aceitado o Termo de Consentimento Esclarecido através da primeira pergunta do questionário. Os dados dos participantes foram tratados com ética e respeito conforme a Resolução 466/2012 do Ministério da Saúde (Brasil, 2012).

\section{Resultados e Discussão}

\subsection{Dados gerais}

Para o presente estudo, foi realizada uma pesquisa on-line sobre idosos usuários de anti-hipertensivos. O questionário foi respondido pelo próprio público-alvo e/ou cuidadores e familiares. Entre os 111 entrevistados, 55,85\% (62) afirmaram apresentar doença cardiovascular, sendo que 2,7\% (3) não informaram o medicamento utilizado, por falta de conhecimento por parte do cuidador ou familiar e/ou do próprio paciente que não sabe o medicamento que utiliza; e 0,9\% (1) informou que não utiliza nenhum medicamento. Houve $21,62 \%$ (24) de respostas negando a doença cardiovascular, porém em uso de antihipertensivos.

Ressaltamos que foram aceitas respostas de cuidadores e familiares, pois essas respostas, além de aumentar o acervo de conteúdo para a pesquisa, assemelham-se à realidade, em que idosos podem não ter capacidade de manter o tratamento sozinhos e realizarem o ato de medica-se com o auxílio de alguém próximo (Didone et al., 2021). Essas respostas também contribuíram para ter um alcance maior de resultados, visto que muitos idosos não têm acesso ao conteúdo on-line.

Na Figura 1 apresenta-se a quantidade de entrevistados de acordo com o respectivo sexo, dos quais 22,52\% (25) homens e 50,45\% (56) mulheres estão em uso de anti-hipertensivo. 
Figura 1. Gênero dos idosos que responderam o questionário.

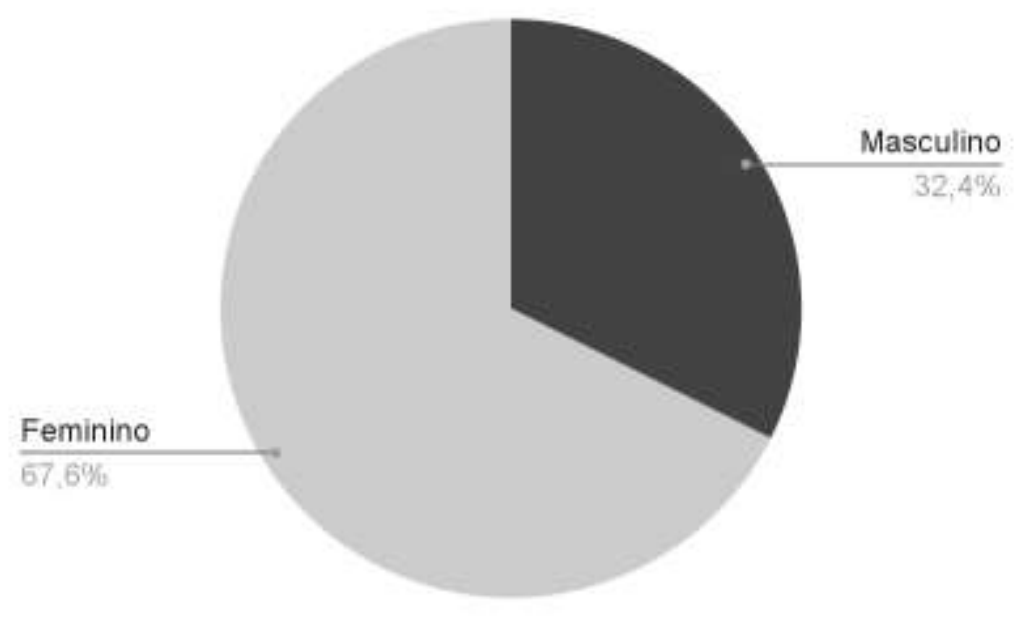

Fonte: Autores (2021).

A faixa etária com maior número de pacientes foi a de 60 a 70 anos, diminuindo conforme a idade aumenta, sendo representado na Figura 2.

Figura 2. Faixa etária dos idosos que responderam o questionário.

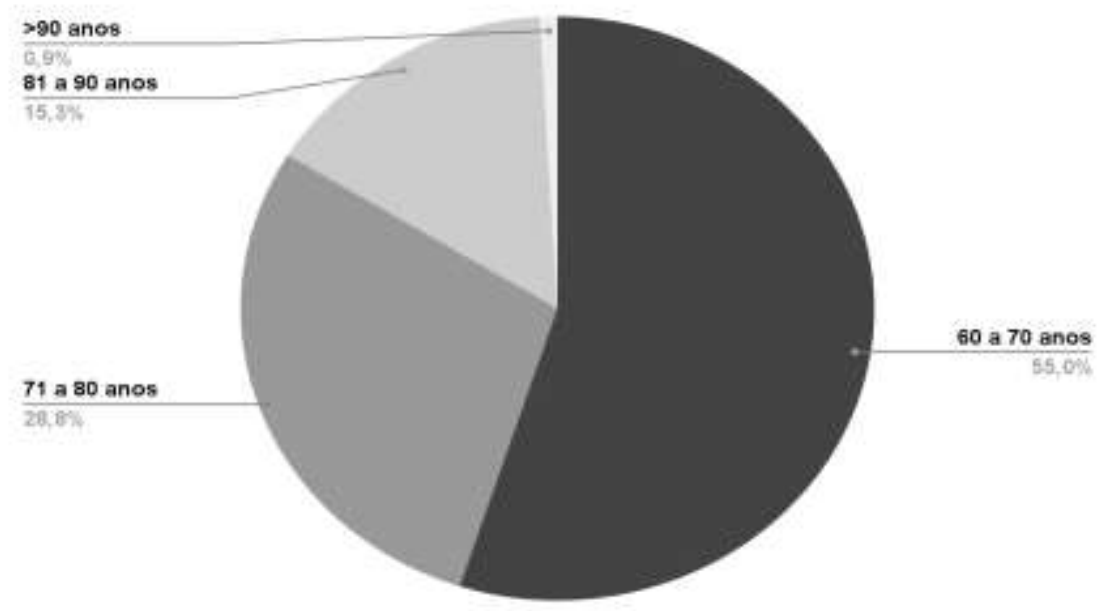

Fonte: Autores (2021).

Dentre os 111 entrevistados, 81 estão em uso de anti-hipertensivo. Na Figura 3, é possível observar a porcentagem dos anti-hipertensivos utilizados por estes 81 pacientes referente às seguintes classes farmacológicas: bloqueador do receptor de angiotensina (BRA), diuréticos tiazídicos, inibidor da enzima conversora de angiotensina (iECA), bloqueadores dos canais de cálcio (BCC), bloqueadores seletivos de beta 1, bloqueadores não seletivos, diuréticos poupadores de potássio e diurético de alça. 
Figura 3. Frequência de uso de anti-hipertensivos por idosos, de acordo com as classes farmacológicas (ordem decrescente).

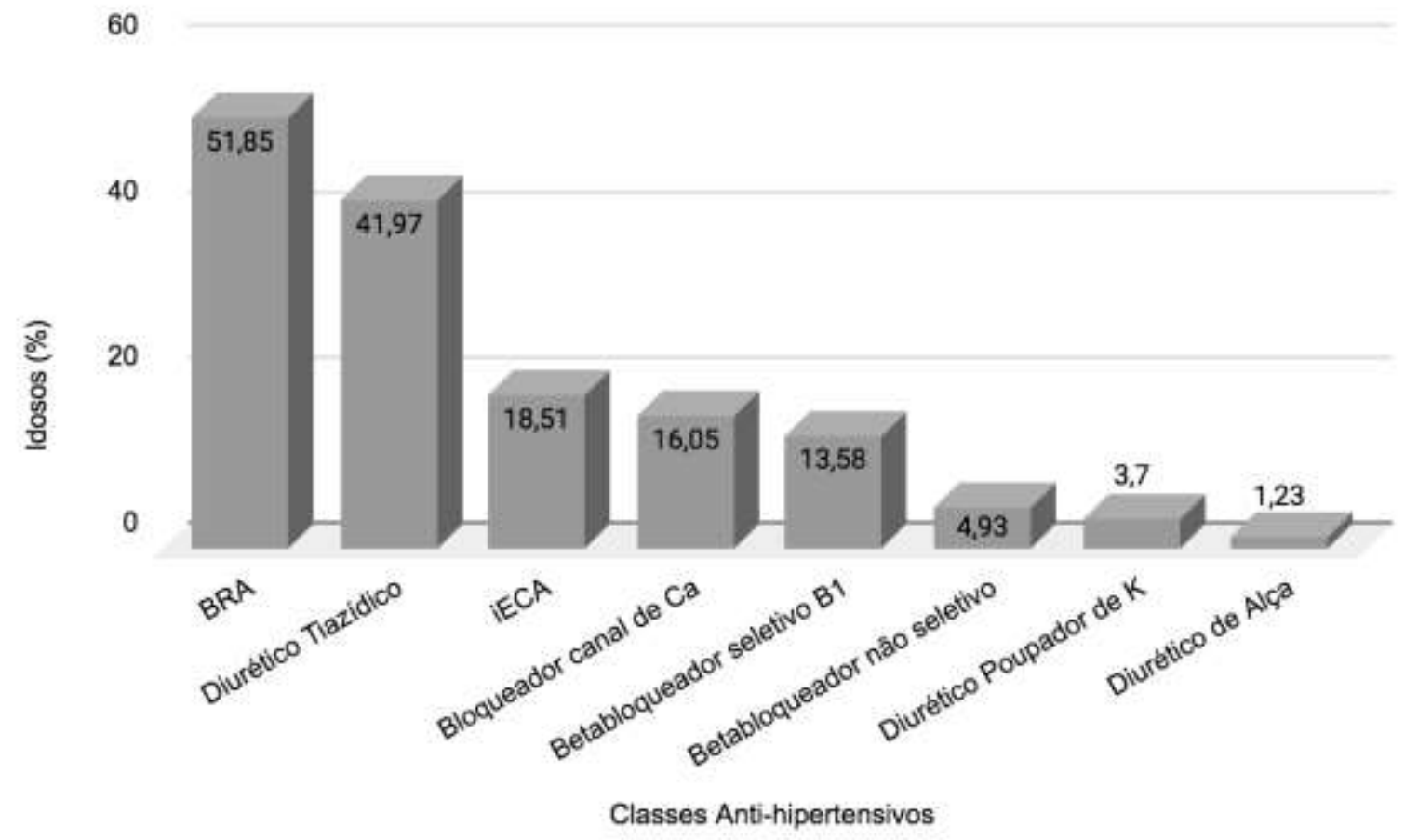

Fonte: Autores (2021).

Dentre os anti-hipertensivos utilizados no tratamento como monoterapia e combinado, 56,79\% (46) dos pacientes estão em monoterapia; e 43,2\% (35) estão em terapia combinada.

\subsection{Pacientes Hipertensos}

Como podemos observar na pesquisa, 51,85\% (42) dos pacientes utilizam BRA; 41,97\% (34) utilizam diuréticos tiazídicos; 18,51\% (15) utilizam iECA; 16,05\% (13) utilizam BCC. Sendo os quatro medicamentos mais utilizados pelos idosos de acordo com os resultados.

Segundo as Diretrizes Brasileiras de Hipertensão Arterial (Malachias et al., 2016), existem três estágios dentro do diagnóstico de hipertensão. O indivíduo entra no quadro de pré-hipertensão e se negligenciado entram para o Estágio 1 podendo evoluir para o Estágio 2 e para o Estágio 3. Na Figura 4, constam os valores de pressão arterial (PA) para cada estágio, incluindo a pré-hipertensão. 
Figura 4. Estágios da hipertensão de acordo com o nível de pressão arterial.
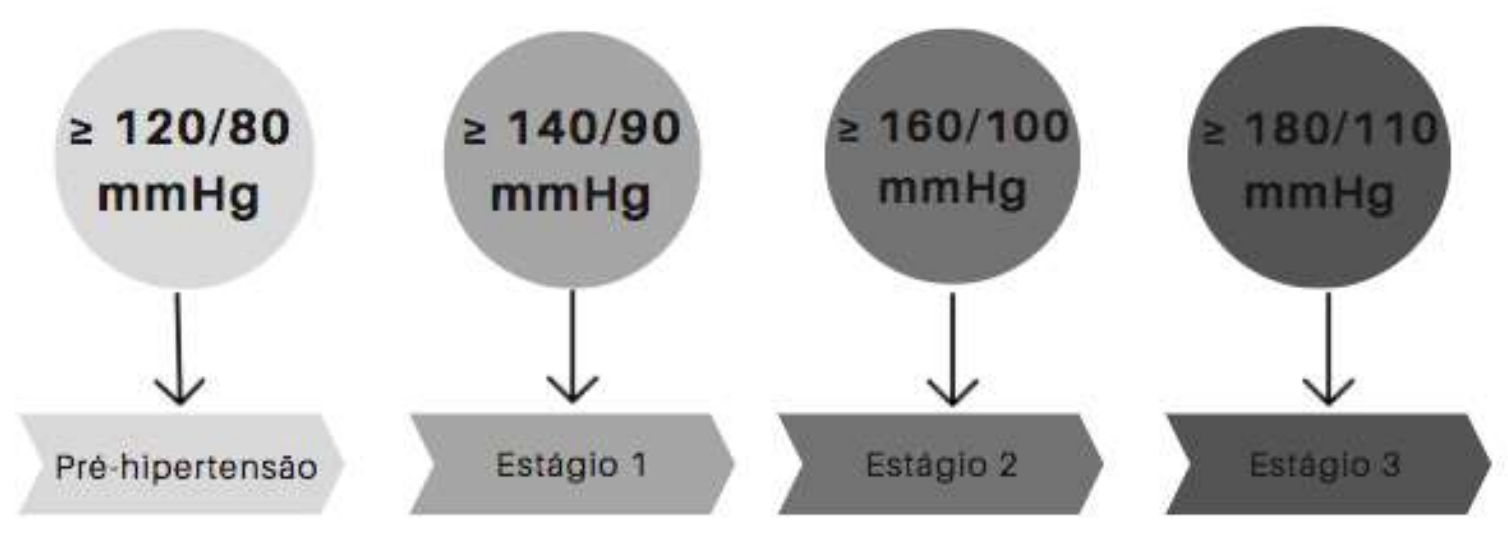

Fonte: Adaptado de (Malachias et al., 2016).

A indicação para o início do tratamento farmacológico, além da terapia não medicamentosa, ocorre quando o paciente apresenta a PA com valores acima dos 140/90 mmHg, ou seja, no Estágio 1 da doença.

Quando ainda no Estágio 1 da doença, mas apresentando um risco cardiovascular baixo/moderado, é indicado o tratamento farmacológico em monoterapia, já quando associado a risco cardiovascular alto, é recomendada uma combinação medicamentosa (Malachias et al., 2016). Portanto, a maioria dos idosos participantes provavelmente ainda está no Estágio 1 da doença, já que 56,79\% (46) estão em monoterapia e somente 43,2\% (35) em terapia combinada.

Tanto nas Diretrizes Brasileiras (Malachias et al., 2016) quanto nas Diretrizes Americanas e Internacionais de Hipertensão Arterial (Weber et al., 2014), existem quatro classes de preferência a serem usadas tanto na monoterapia, quanto na terapia de associação: diuréticos tiazídicos, iECA, BRA e BCC. Portanto, é esperado que os mais utilizados estejam dentro dessas classes.

Para os Estágios 2 e 3 da doença associados a qualquer risco cardiovascular, é indicada a terapia com associação medicamentosa (Malachias et al., 2016). Embora na maioria dos casos de hipertensão seja necessário mais de um medicamento para o controle total da pressão (Weber et al., 2014), a pesquisa demonstrou que a maioria dos idosos está com a hipertensão controlada com apenas a monoterapia.

Na pesquisa, embora a classe mais utilizada em monoterapia sejam os BRAs, com 51,85\% (42) dos pacientes em uso, os diuréticos são a classe de fármacos considerada mais efetiva para a diminuição de risco cardiovascular (Malachias et al., 2016), o que é uma grande preocupação quando se trata da escolha. Isso acontece porque mesmo sendo os diuréticos mais indicados, é normal que pacientes não se adaptem a ele e seja indicado a troca do medicamento ou alteração de posologia. Além disso, a escolha do medicamento também pode variar de acordo com o sexo, a idade, a raça e outros fatores clínicos associados ao paciente (Weber et al., 2014). Se o aumento de posologia ou troca do medicamento ainda não atingir a meta terapêutica desejada, ou causar efeitos adversos, entram as associações entre hipertensivos (Malachias et al., 2016).

Na pesquisa, podemos notar que as associações mais comuns são as entre diuréticos tiazídicos e BRA 17,28\% (14). Isso porque é indicado que sejam associados medicamentos com mecanismos de ação diferentes (Malachias et al., 2016). São encorajadas escolhas como diuréticos e iECA, diuréticos e BCC, BCC e iECA, e assim por diante. Entre estas, só não costuma ser indicada a associação de iECA com BRA, já que apresenta grande número de efeitos adversos e não diminui como desejado o risco cardiovascular (Malachias et al., 2016), embora tenha sido observado que 1,23\% (1) utiliza essa associação. 
É comum também a escolha de medicamentos que sejam multifuncionais e que consigam ser utilizados em diferentes estágios da doença, sozinhos ou associados a algum medicamento, ou que tenham dois compostos na mesma formulação (Weber et al., 2014), lembrando que o fármaco de escolha pode sempre mudar dependendo da comorbidade de um paciente.

Na pesquisa, também foi questionado se os entrevistados possuíam alguma outra patologia. Os resultados com mais relevância apresentados foram: 35,71\% (30) com diabetes; 27,38\% (23) com alteração no colesterol (dislipidemia); e 10,71\% (9) com gastrite, além de outras patologias com menor relevância para o estudo. Isso é relevante, uma vez que de acordo com as Diretrizes Brasileiras de Hipertensão Arterial (Malachias et al., 2016), a hipertensão pode ser agravada por fatores de risco como obesidade, dislipidemia, intolerância à glicose e diabetes mellitus (DM). Ainda assim, 32,14\% (27) dos idosos não indicaram nenhuma dessas patologias, podendo ser por falta de conhecimento ou de outra patologia.

Além disso, segundo as Diretrizes Internacionais da Hipertensão Arterial (Weber et al., 2014), as comorbidades apresentadas também podem alterar a PA, uma vez que é comum a utilização de medicamentos como antidepressivos, AINES, contraceptivos orais combinados, esteroides, entre outros no tratamento.

\subsection{Manuseio geral dos medicamentos}

Muitos fatores causam a não adesão farmacológica dos pacientes, como preocupações relacionadas ao custo do tratamento; esquecimento dos horários de utilização, muitas vezes por serem doses múltiplas; baixo nível intelectual; falta de sintomas; e eventos adversos ou dificuldade para conseguir vagas de consulta médica. Pacientes que possuem uma rede de apoio de familiares e amigos são mais propensos a realizar o tratamento de forma mais correta e quanto maior o nível de adesão ao tratamento menos despesas médicas são apresentadas pelos pacientes (Cardoso et al., 2021).

Cerca de 55\% dos pacientes idosos não aderem ao tratamento farmacológico com anti-hipertensivos por fatores como comorbidade, idade, baixo nível socioeconômico, limitações físicas, entre outros. Além disso, a falta de orientação ou orientação incorreta podem causar uma falha na ingestão do medicamento, bem como o corte indevido de comprimidos ou abertura de cápsulas e outros erros como o armazenamento incorreto (Jankowska-Polańska et al., 2016). Nossa pesquisa indicou, de forma geral, informações sobre ingestão, corte de comprimidos ou abertura de cápsulas e armazenamento de antihipertensivos por idosos, como representado na Figura 5. 
Figura 5. Manuseio de anti-hipertensivos por indivíduos com mais de 60 anos.

Ingestăo
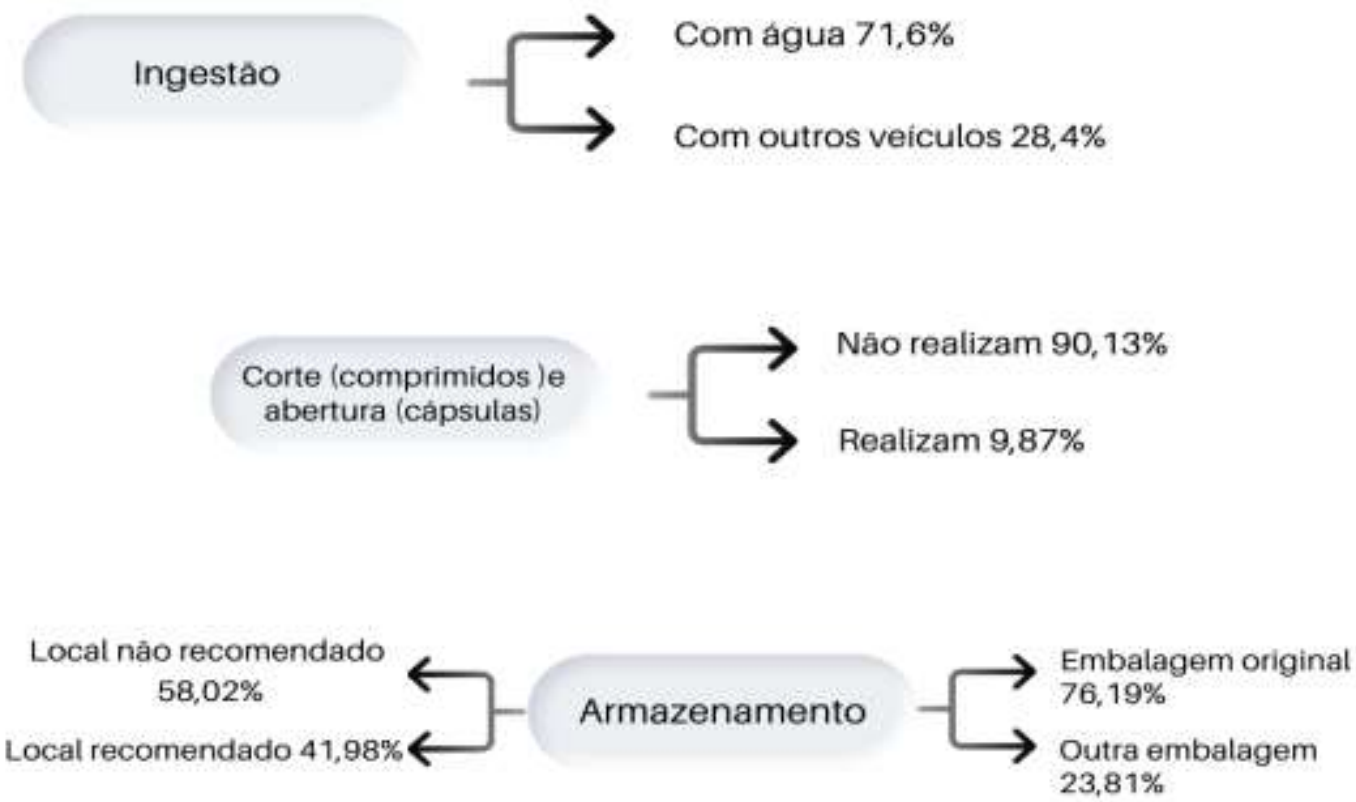

Fonte: Autores (2021).

A Resolução 328/99 Ministério da Saúde informa sobre os requisitos para dispensação de medicamentos em drogarias e farmácias visando boas práticas de assistência farmacêutica, sendo de responsabilidade e atribuição do farmacêutico avaliar a prescrição médica (Brasil, 1999). É papel do farmacêutico detectar a necessidade de medidas preventivas ou corretivas para um tratamento mais eficaz e seguro, prestando um serviço individual e humano ao paciente em uso de anti-hipertensivos, que em sua maioria são idosos (Penha et al., 2021).

\subsubsection{Ingestão}

Os resultados referentes à ingestão de medicamentos foram 71,6\% (58) administrados somente com água; 7,4\% (6) com café e leite; 4,93\% (4) somente com suco; 3,7\% (3) com suco e refrigerante; 3,7\% (3) não apresentaram respostas; $2,46 \%$ (2) somente com refrigerante; $2,46 \%$ (2) somente com café; $1,23 \%$ (1) afirmou administrar com álcool, suco, refrigerante e café; $1,23 \%$ (1) afirmou administrar com chá, café e leite; e 1,23\% (1) administram sem auxílio de nenhum líquido ou alimento.

O veículo utilizado para a ingestão de comprimidos e cápsulas pode acarretar interação entre droga-nutriente, da qual pode influenciar no tratamento de maneira positiva ou negativa. A falta de conhecimento e de orientação farmacêutica pode gerar danos severos à terapêutica como a alteração da farmacodinâmica do fármaco, toxicidade, deficiências nutricionais e reações adversas. Apesar disso, em alguns casos, essa interação pode ser benéfica, podendo auxiliar na biodisponibilidade e absorção do fármaco, além de ajudar a evitar danos ao sistema gastrointestinal (Koziolek et al., 2019).

Em uma ordem decrescente dos fármacos mais utilizados na pesquisa, em relação aos BRAs não foram encontradas interações com alimentos significativos. Os diuréticos tiazídicos, especificamente a Hidroclorotiazida e Clortalidona, quando ingeridos com leite, evitam os danos ao trato gastrointestinal e estimulam a absorção do fármaco. Os iECAs, especificamente o Captopril e Enalapril, quando ingeridos com alimentos ricos em potássio pode causar hipercalemia, e quando ingeridos com 
alimentos ricos em zinco pode resultar em hipogeusia e, consequentemente, alteração do estado nutricional (Pereira et al., 2020).

Dos BBCs, a Nifedipina apresentou interação com o suco de toranja, aumentando a biodisponibilidade do fármaco, risco de efeitos colaterais e hipotensão; o Verapamil apresentou interação com o café, aumentando a concentração sérica da cafeína e aumentando a estimulação do sistema nervoso central, e com o suco de toranja, aumentando a biodisponibilidade do fármaco, risco de efeitos colaterais e hipotensão; e o Diltiazem apresentou interação com o café, aumentando a concentração sérica da cafeína e aumentando a estimulação do sistema nervoso central (Pereira et al., 2020). De todos os idosos que utilizam anti-hipertensivos, somente 2,46\% (2) responderam fazer uso de algum BBC junto com café, podendo resultar em alguma dessas interações medicamentosas.

Dos betabloqueadores seletivos para B1, especificamente o Atenolol, apresentam interação com o suco de de laranja, diminuindo a absorção do fármaco, e com o suco de maçã, diminuindo a biodisponibilidade e eficácia do fármaco e aumentando a concentração luminal da droga. Dos betabloqueadores não seletivos, especificamente o Propanolol, quando ingeridos com alimentos hiperproteicos auxiliam na biodisponibilidade do fármaco (Pereira et al., 2020).

Quanto aos diuréticos poupadores de potássio, especificamente a Espironolactona, é benéfico sua ingestão com alimentos, para diminuir a irritação do trato gastrointestinal, porém quando ingeridos com alimentos ricos em potássio podem causar hipercalemia. Em relação aos diuréticos de alça, especificamente a Furosemida, quando ingeridos com alimentos em geral ou leite, causam diminuição do desconforto do trato gastrointestinal e retardam a absorção do fármaco, podendo desencadear uma diminuição da resposta diurética (Pereira et al., 2020).

Na pesquisa, um dos entrevistados afirmou fazer a ingestão do anti-hipertensivo com álcool. Uma das alternativas que pode justificar tal resultado pode estar ligado a não informação do paciente ou por elitismo dependente.

Em idosos, o uso concomitante de álcool com agentes cardiovasculares aumenta o risco de hipotensão (Holton et al., 2017). Além disso, o consumo de álcool pode agravar a patologia, ser responsável pela não adesão ao tratamento e deixar os órgãos-alvo mais vulneráveis (Rodrigues Júnior et al., 2019).

\subsubsection{Corte e abertura de cápsulas}

Os pacientes em uso de anti-hipertensivos foram questionados se realizavam abertura da cápsula ou corte do comprimido utilizado: 9,87\% (8) pacientes informaram que realizam o corte do comprimido, sendo 1,23\% (1) triturado, 2,47\% (2) com cortador e 6,17\% (5) com faca. Já em relação a abertura de cápsula, 1,23\% (1) paciente afirmou realizar esse procedimento para ingestão do conteúdo com água.

O processo de partição de um comprimido é uma alteração física no medicamento que pode causar problemas de estabilidade, possibilidade de irritação ao trato gastrointestinal e de intoxicação e, em caso de medicamentos que possuam liberação modificada, podem ter seus mecanismos afetados. A partição pode ser orientada em casos de comprimidos sulcados, que foram avaliados por testes de qualidade da possibilidade de partição (Teixeira et al., 2016). A partição destes comprimidos sulcados também é abordada na maioria das farmacopeias, que estudam a uniformidade do corte, variação de peso e a perda de massa do fármaco (FDA, 2013).

A utilização de utensílios não apropriados como a faca, por exemplo, têm influência direta no aumento da quantidade de princípio ativo ingerida quando comparado com o partidor de comprimidos (Auricchio et al., 2011). Não é possível ter precisão com a dose de comprimidos partidos em relação à formulação íntegra, embora em um estudo realizado em 2011, que avaliou o teor de Atenolol em comprimidos divididos, mostrou que não houve diferenças significativas quando cortados com facas ou com o divisor de comprimidos (Auricchio et al., 2011). 
Dos pacientes que realizam, o corte de comprimidos, metade informou que foram orientados por um profissional de saúde e a outra metade não. O paciente que indicou realizar abertura de cápsula afirmou ter sido orientado por um profissional de saúde. Muitas vezes a orientação de corte do comprimido ocorre por tentativa de alteração na dose ou baixo nível socioeconômico, mas a realização do corte não tem garantia de dose adequada e compromete a estabilidade do produto (BorjaOliveira 2013).

A prática de triturar o comprimido para uso do paciente é comum em pacientes com dificuldade de deglutição, como idosos, que não querem realizar o uso do medicamento ou pacientes com doenças mentais graves, podendo em alguns casos após a trituração misturar o medicamento com algum alimento ou bebida para a administração. Alguns casos são indicados pelo profissional de saúde, quando discutido e concluído como melhor forma de administração (Haw and Stubbs 2010). Esse tipo de prática também pode se assemelhar a de abertura de cápsulas para a ingestão.

Muitos pacientes com o passar dos anos vão perdendo o controle de seus movimentos, e no momento da partição dos comprimidos podem não ter a destreza necessária para a divisão, e mesmo quando orientados por um profissional de saúde para realização da partição, resulta em uma divisão desigual, o que pode causar uma subdosagem ou sobredosagem devido a variação de peso e uniformidade (Jacques and Alexandridis 2019).

Para o armazenamento da parte restante do comprimido, deve-se ter atenção e cuidado, para manter a temperatura e a umidade ideal para o medicamento. Porém, a orientação é manter na embalagem original e partir um comprimido de cada vez, pois após a partição e em atrito com outros comprimidos pode causar maior friabilidade (Teixeira et al., 2016).

\subsubsection{Armazenamento}

Sobre o local de armazenamento do medicamento, 44,44\% (36) afirmaram guardar na cozinha; $40,74 \%$ (33) no quarto; $12,34 \%$ (10) no banheiro; $1,23 \%$ (1) na bolsa; e 1,23\% (1) na sala. Além disso, 77,78\% (63) afirmaram utilizar a embalagem original do medicamento e $22,22 \%$ (18) afirmaram fazer uso de outro recipiente para armazenamento do medicamento que não seja a embalagem original.

Em um estudo de corte transversal realizado com idosos residentes da Holanda, o resultado do local mais armazenado também é a cozinha, seguido da sala de estar e quarto (Vlieland et al., 2018). Embora seja o resultado mais comum, não é recomendado o armazenamento de medicamentos na cozinha, uma vez que ele está mais suscetível ao aumento e diminuição de temperatura e umidade, que podem alterar a estabilidade do medicamento, o que pode comprometer o efeito terapêutico. Embora seja possível essa alteração, levando a deterioração do fármaco, outro estudo observacional descritivo demonstrou baixos sinais macroscópicos de deterioração nos medicamentos armazenados de forma incorreta em residências brasileiras (Paolinelli et al., 2021).

Em relação ao armazenamento incorreto destes medicamentos, um estudo de corte transversal realizado em 2014, no Brasil, demonstrou que a idade estava ligada a esses erros de armazenamento, indicando que idosos estavam mais propensos a cometer esse tipo de erro (Martins et al., 2017).

Além disso, polifarmácia também é algo comum em idosos, o que pode aumentar os obstáculos com medicamentos, como armazenamentos incorretos, ingestão medicamentos que já passaram da data de vencimento e confusão na ingestão.

A polifarmácia também pode acabar resultando no uso de organizadores, porém estes podem prejudicar a estabilidade do medicamento, já que ficam em exposição direta à luz (em relação a medicamentos fotossensíveis), e também podem confundir o usuário em relação a qual medicamento está sendo ingerido, a data de validade e a dosagem, uma vez que retirados da sua embalagem original não possui as informações necessárias de cada um. O que pode auxiliar é o corte de parte da cartela que envolve o comprimido (blister), mantendo-o na embalagem original e ajudando na identificação. Porém, ao se tratar de idosos, a identificação pode ficar pequena e difícil de ser visualizada (Borja-Oliveira 2013). 
Portanto, o armazenamento deve ser realizado conforme a necessidade do medicamento referente à temperatura, umidade e em sua embalagem primária, para a garantia de qualidade do medicamento (Vlieland et al., 2018).

\section{Conclusão}

O acompanhamento e tratamento farmacológico da hipertensão arterial em idosos é um grande desafio que envolve desde a orientação correta para administração até o armazenamento dos medicamentos. O profissional farmacêutico está envolvido diretamente nesse processo, devendo orientar adequadamente o doente e/ou seu acompanhante para que o resultado da terapia medicamentosa ocorra da maneira esperada. Este estudo demonstrou que a maioria dos idosos em uso dos antihipertensivos está em monoterapia, sendo a associação mais utilizada é de BRA com diuréticos tiazídicos. Em relação ao manuseio, o uso está sendo correto na maioria, como administração do medicamento com água. Além disso, poucos casos de prática de corte de comprimidos e abertura de cápsulas foram detectados. Quanto ao armazenamento do medicamento na embalagem primária, a maioria está em conformidade com as recomendações. Mas ao se tratar do local de armazenamento na residência, locais com temperatura e umidade fora dos solicitados em bula estão em maioria, sendo eles a cozinha e o banheiro. Embora a pesquisa tenha apresentado resultados positivos, os erros identificados poderiam ser facilmente evitados com a orientação correta e constantemente reforçada dos profissionais de saúde. Embora o trabalho explore uma classe terapêutica muito consumida pela polulação idosa, faz-se necessários mais estudos para avaliar os hábitos relacionados a outras classes de medicamentos, como os empregados para a diabetes, por exemplo, que também são amplamente utilizados e também requerem atenção quanto ao seu correto uso e manuseio.

\section{Referências}

Auricchio, M. T., Yano, H. M., Santos, A. P., \& Bugno, A. (2011). Avaliação do teor de Atenolol em comprimidos divididos com faca caseira e aparelho cortador. Acta Paulista de Enfermagem, 24(1), 74-79.

Borja-Oliveira, C. R. de. (2013). Pill organizers and pill cutters: risks and limitations. Revista de Saúde Pública, 47(1), $123-127$.

Brasil. (1999) Ministério da Saúde. Resolução n 328, de 22 de julho de 1999. Dispõe sobre requisitos exigidos para a dispensação de produtos de interesse à saúde em farmácias e drogarias. https://www.cff.org.br/userfiles/file/resolucao_sanitaria/328.pdf

Brasil. (2012) Ministério da Saúde. Resolução n 466, de 12 de dezembro de 2012. Diretrizes e normas regulamentadoras de pesquisas envolvendo seres humanos, 150 Diário Oficial da União [da] República Federativa do Brasil 59 (2013). https://bvsms.saude.gov.br/bvs/saudelegis /cns/2013/res0466_12_12_2012.html

Burnier, M., Polychronopoulou, E., \& Wuerzner, G. (2020). Hypertension and Drug Adherence in the Elderly. Frontiers in Cardiovascular Medicine, 7, 49.

Cardoso, G. da S., Sousa Neto, B. P. de, Magalhães, N. A., Cardoso, L. da S., Costa, G. O. P. da, Ribeiro, A. M. N., Jatobá, D. N. V., Mariano, S. C. B., Cruz, F. C. da, Pinheiro, D. M., Castro, M. C. de O., Paz, M. I., Araújo, D. S. de, \& Jansen, R. C. S. (2021). Fatores que interferem na adesão ao tratamento farmacológico em idosos hipertensos. Research, Society and Development, 10(2), e17510212352.

Center for Drug Evaluation, \& Research. (2021). About FDA Guidances. http://www.fda.gov/Drugs/GuidanceComplianceRegulator yInformation/Guidances/default.htm

Didone, T. V. N., Melo, D. O. de, Lopes, L. da C., \& Ribeiro, E. (2021). Conhecimento inadequado sobre medicamentos prescritos e seus preditores em pacientes muito idosos e seus cuidadores. Revista Brasileira de Geriatria e Gerontologia, 23(3). https://doi.org/10.1590/1981-22562020023.200193

Gill, D., Spain, M., \& Edlund, B. J. (2012). Crushing or splitting medications: unrecognized hazards. Journal of Gerontological Nursing, 38(1), 8-12.

Haw, C., \& Stubbs, J. (2010). Administration of medicines in food and drink: a study of older inpatients with severe mental illness. International Psychogeriatrics / IPA, 22(3), 409-416.

Holton, A. E., Gallagher, P., Fahey, T., \& Cousins, G. (2017). Concurrent use of alcohol interactive medications and alcohol in older adults: a systematic review of prevalence and associated adverse outcomes. BMC Geriatrics, 17(1), 148.

Jacques, E. R., \& Alexandridis, P. (2019). Tablet Scoring: Current Practice, Fundamentals, and Knowledge Gaps. NATO Advanced Science Institutes Series E: Applied Sciences, 9(15), 3066.

Jankowska-Polańska, B., Dudek, K., Szymanska-Chabowska, A., \& Uchmanowicz, I. (2016). The influence of frailty syndrome on medication adherence among elderly patients with hypertension. Clinical Interventions in Aging, 11, 1781-1790. 
Jáuregui-Garrido, B., \& Jáuregui-Lobera, I. (2012). Interactions between antihypertensive drugs and food. Nutricion Hospitalaria: Organo Oficial de La Sociedad Espanola de Nutricion Parenteral Y Enteral, 27(6), 1866-1875.

Koziolek, M., Alcaro, S., Augustijns, P., Basit, A. W., Grimm, M., Hens, B., Hoad, C. L., Jedamzik, P., Madla, C. M., Maliepaard, M., Marciani, L., Maruca, A., Parrott, N., Pávek, P., Porter, C. J. H., Reppas, C., van Riet-Nales, D., Rubbens, J., Statelova, M., \& Corsetti, M. (2019). The mechanisms of pharmacokinetic food-drug interactions - A perspective from the UNGAP group. European Journal of Pharmaceutical Sciences: Official Journal of the European Federation for Pharmaceutical Sciences, 134, 31-59.

Malachias, M. V. B., Plavnik, F. L., \& Machado, C. A. (2016). 7ª Diretriz Brasileira de Hipertensão Arterial: Capítulo 1-Conceituação, Epidemiologia e Prevenção Primária. De Cardiologia. https://www.scielo.br/j/abc/a/LtmRBQ7ZnJ88SQxL64yFRyy/?format=pdf\&lang=pt

Martins, R. R., Farias, A. D., Oliveira, Y. M. da C., Diniz, R. D. S., \& Oliveira, A. G. (2017). Prevalence and risk factors of inadequate medicine home storage: a community-based study. Revista de Saúde Pública, 51, 95.

Nascimento, L.F., Cavalcante, M.M.D. (2018). Abordagem quantitativa na pesquisa em educação: investigações no cotidiano escolar. Revista Tempos e Espaços em Educação, 11(25), 251-262.,

Nobre de Menezes, T., Tôrres Oliveira, E. C., Abreu Tavares Sousa Fischer, M., \& Esteves, G. H. (2016). Prevalência e controle da hipertensão arterial em idosos: um estudo populacional. Revista Portuguesa de Saúde Pública, 34(2), 117-124.

Paolinelli, J. P. V., Morais, D. B., Oliveira, W. N., Fernandes, M. R., Rocha, R. S., Figueiredo, R. C., Baldoni, A. O., \& Pereira, M. L. (2021). Macroscopic signs of deterioration of medicines in home stocks. Research, Society and Development, 10(7), e28910716517-e28910716517.

Penha, B. C. M., Marques, G. P., \& Rodrigues, K. M. R. (2021). Acompanhamento farmacoterapêutico do paciente idoso com hipertensão arterial em população brasileira: achados de revisão sistemática/ Pharmacotherapeutic follow-up of the elderly patient with hypertension in the brazilian population: findings from a systematic review. Brazilian Journal of Health Review, 4(3), 11412-11425.

Pereira, M. T. L., Queiroz, M. P., de Menezes Santos Bertozzo, C. C., Barbosa, M. Q. E., da Silva Campos, R., \& Pinto, M. L. M. (2020). Drug-nutrient interactions of antihypertensives and antidiabetics prescribed at Alcides Carneiro University Hospital. Research, Society and Development, 9(7), e35973802e35973802

Rodrigues Júnior, F. A., Alencar, M. M. F., Menezes, J. J. de, \& Galvão, P. V. M. (2019). A relação entre o uso de álcool e a não adesão ao tratamento em pacientes hipertensos. Multidisciplinary Reviews, 2(1), e2019009.

Sousa, A. L. L., Batista, S. R., Sousa, A. C., Pacheco, J. A. S., Vitorino, P. V. de O., \& Pagotto, V. (2019). Hypertension Prevalence, Treatment and Control in Older Adults in a Brazilian Capital City. Arquivos Brasileiros de Cardiologia, 112(3), 271-278.

Teixeira, M. T., Sá-Barreto, L. C. L., Silva, D. L. M., \& Cunha-Filho, M. S. S. (2016). Panorama dos aspectos regulatórios que norteiam a partição de comprimidos. Revista panamericana de salud publica = Pan American journal of public health, 39(6), 372-377.

Vlieland, N. D., van den Bemt, B. J. F., Bekker, C. L., Bouvy, M. L., Egberts, T. C. G., \& Gardarsdottir, H. (2018). Older Patients' Compliance with Drug Storage Recommendations. Drugs \& Aging, 35(3), 233-241.

Weber, M. A., Schiffrin, E. L., White, W. B., Mann, S., Lindholm, L. H., Kenerson, J. G., Flack, J. M., Carter, B. L., Materson, B. J., Ram, C. V. S., Cohen, D. L., Cadet, J.-C., Jean-Charles, R. R., Taler, S., Kountz, D., Townsend, R. R., Chalmers, J., Ramirez, A. J., Bakris, G. L., \& Harrap, S. B. (2014). Clinical practice guidelines for the management of hypertension in the community: a statement by the American Society of Hypertension and the International Society of Hypertension. Journal of Clinical Hypertension, 16(1), 14-26. 